\title{
GIA 2011: Genomics in Aquaculture 2011 Symposium
}

\author{
Elena Sarropoulou • Jorge Fernandes • \\ Zhanjiang John Liu
}

Published online: 8 July 2012

(C) Springer Science+Business Media, LLC 2012

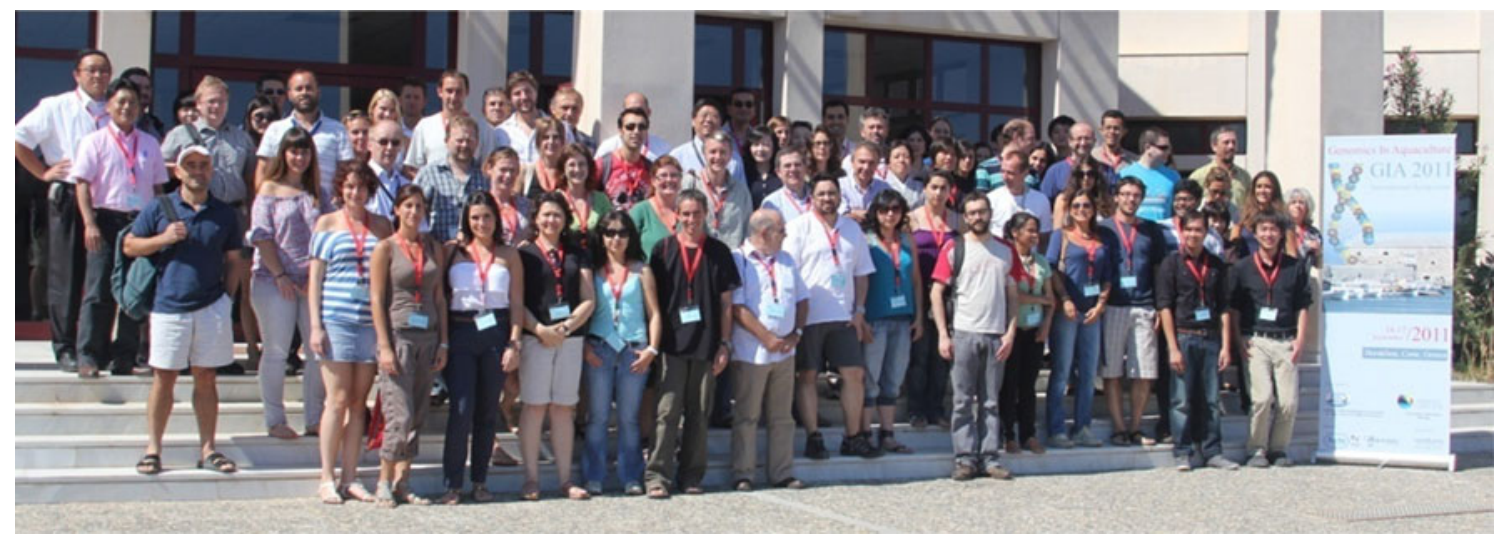

Genomics, although a relatively young branch of science, has had a tremendous scientific, social, and economic impact in the world. Aquaculture genomics research started around 15 years ago, but progress at the beginning was slow

\author{
E. Sarropoulou $(\square)$ \\ Institute of Marine Biology, Biotechnology and Aquaculture, \\ Hellenic Centre for Marine Research, \\ P.O. Box 2214, 71003 Heraklion, Greece \\ e-mail: sarris@her.hcmr.gr \\ E. Sarropoulou \\ e-mail: sarris@hcmr.gr

\section{J. Fernandes} \\ Marine Genomics Research Group, Faculty of Biosciences and \\ Aquaculture, University of Nordland, \\ Bodø, Norway \\ e-mail: Jorge.fernandes@uin.no
}

\section{Z. J. Liu}

The Fish Molecular Genetics and Biotechnology Laboratory, Department of Fisheries and Allied Aquacultures, Program of Cell and Molecular Biosciences, Aquatic Genomics Unit, Auburn University,

Auburn, AL 36849, USA

e-mail: Liuzhan@auburn.edu due to limited funding. However, this situation has been changing due to technological advances that are accessible to aquaculture researchers. Among many of the technological advances, the next-generation sequencing technologies have had the largest impact on aquaculture genomics. Nowadays the sequencing cost is dropping rapidly, enabling studies of structure, expression, and function of the entire genome in a wide variety of aquaculture species. Along with the major progress made in aquaculture genomics in the last several years, there are major challenges in dealing with the huge amount of data. Large datasets in multiples of terabytes themselves pose challenges in data processing, analysis, and storage. More importantly, how to draw biological information useful for aquaculture out of the huge datasets is a tremendous challenge. Facing such challenges, the series of the Genomics in Aquaculture (GIA) symposium was born.

The first GIA symposium was held in 2009. This symposium was funded by the Research Council of Norway (refs. 192126 and 190350) and organized by the University of Nordland (Norway) in collaboration with the Hellenic Centre for Marine Research (Heraklion, Greece) and the National Institute of Nutrition and Seafood Research (Norway). Its remarkable success and the 
requests of many participants inspired us to organize the second Genomics in Aquaculture Symposium in 2011 (GIA 2011). The host of the symposium was the Institute of Marine Biology, Biotechnology, and Aquaculture of the Hellenic Centre for Marine Research in Heraklion, Crete, Greece.

The scientific coordination was by the University of Nordland (Norway) and by the Institute of Marine Biology, Biotechnology, and Aquaculture of the Hellenic Centre for Marine Research (Greece). We are thankful to Prof Kiron Viswanath (University of Nordland) for his contribution, including the design of the logo presented in the cover of this special issue. The general organization of GIA 2011 was by Georgia Tsiskaki at CREATEVENTS S.A. (Greece), to whom we are particularly grateful.

The GIA 2011 had the participation of ca. 100 delegates from 17 countries, 48 speeches including 6 leading experts in genomics in aquaculture. Presentations covered a wide range of the main aquaculture species, the two economically important species in the Mediterranean, the gilthead sea bream (Sparus aurata) and the European sea bass (Dicentrarchus labrax); important aquaculture species of Northern Europe like the Atlantic salmon (Salmo salar); the Atlantic cod (Codus morhua); important freshwater fish species like the rohu carp (Labeo rohita) as well as commercially relevant tuna species.

The studies presented dealt with growth and nutrition, immune and stress response, as well as reproduction and breeding. In addition to the broad range of species and research topics, a wide variety of state-of-the-art technologies were represented. This Special Issue includes some examples of the species, research topics, and technologies in 12 papers. Studies related to sex determination and sex-specific marker were presented by Vinas et al. and Piferrer et al. The former deals with the mapping of sex-specific markers in turbot, whereas the latter reviews genomic approaches to study genetic and environmental influences on fish sex determination and differentiation. Next-generation sequencing was also performed for RNAseq in the rohu carp by Robinson et al., the Atlantic salmon by Micallef et al., and the European sea bass by Sarropoulou et al., addressing polymorphism in expressed sequence tags, gene expression in the skin as defense organ, and gene expression after vaccination of fish, respectively. Related to immune response, the studies of P. Díaz-Rosales et al. and Belén G. Pardo et al. used the microarray technology to identify differentially expressed genes in turbot (Scophthalmus maximus). A microarray approach was also used to study the response of skeletal muscle to lipopolysaccharide in the gilthead sea beam, an important food species in the Mediterranean (Kaitetzidou et al.). Two papers are presented in this issue covering freshwater species: one describing the biology and the progress in genomics in freshwater eels (genus Anguilla) (Minegishi et al.) and the other describing immune responses and effects of feeding restrictions in rainbow trout (Oncorhynchus mykiss) (Wenger et al.). For rearing Atlantic cod larvae, a nucleotide enrichment of live feed protocol and its influence in gene expression are described by Lanes et al. As model fish species in relation to aquaculture species are gaining more and more importance, one manuscript by Vareilles et al. deals with the influence of dietary lysine imbalance in the muscle proteome of zebrafish (Danio rerio).

While this Special Issue publishes only some examples of the research papers presented at GIA 2011, the symposium was clearly a tremendous success. Unlike many genomics meetings that cover so many different species and topics, the GIA symposium provided a platform specifically for aquaculture genomicists and geneticists to interact, discuss, and establish collaborations. We, as the editors of this Special Issue, would like to thank the sponsors, organizers, invited speakers, and all participants for the great success of GIA 2011. We would also like to express our gratitude to Marine Biotechnology for publishing this Special Issue. 